\title{
Analysis of WeChat Subscription Influence Based on Topic Diffusion
}

\author{
Cong Zhang, Beijing University of Posts and Telecommunications, Beijing, China \\ Yuan'An Liu, Beijing University of Posts and Telecommunications, Beijing, China \\ Fan Wu, Beijing University of Posts and Telecommunications, Beijing, China \\ Lidong Zhai, Institute of Computing Technology of the Chinese Academy of Sciences, Beijing, China \\ Hui Lu, Guangzhou University, Guangzhou, China
}

\begin{abstract}
With the average daily growth of 15000 new subscribers, the number of WeChat subscription has broken the 5,800,000 subscribers recently. WeChat subscription's topic influence calculation has been very significant. This article takes Tencent-WeChat subscription platform as the research object and focuses on the influence analysis of topic diffusion. Based on the subscription's temporal characteristics of influence, the parameters of propagation capability, and the similarity weights among different subscriptions with the same topic, the authors propose an analysis model of WeChat subscription influence.
\end{abstract}

\section{KEYWORDS}

Impact Temporal Characteristics, Influence, Topic, WeChat Subscription

\section{INTRODUCTION}

Nowadays, with the rapid development of the Internet, the era of big data is coming. As the essential development of Internet application, sociality is no longer limited to the transmission of information. It has been the integration of communication and business transaction applications. WeChat is one of the most popular social networks today, which has a lot of advantages. For instance, on the one hand, users can publish messages at anytime and anywhere, and on the other hand, the interaction is becoming strong and easy to operate for users, etc. According to the 40th China Internet development statistics report of CNNIC (China Internet Network Information Center) in June 2017, the usage rate of WeChat is $84.3 \%$, which has a great advantage compared with other public applications. WeChat subscription is one of the main routes of WeChat's information dissemination. WeChat official latest news shows that the total number of subscriptions is over 5,800,000 and growing on average 15,000 every day. With the rapid development of WeChat subscription, it has a great theoretical and practical significance to evaluate the influence of WeChat's subscription within the same article topic. When an article is pushed by WeChat subscription spreads rapidly in WeChat, it means that a lot of people read, comment and praise. Finally, it will affect the whole public opinion situation. If the content of subscription's article involves rumors and bad information, it will have the adverse impact on society and lead to public opinion panic.

At present, social impact analysis algorithm is mainly applied in micro-blog. However, in the aspect of the dissemination of relations and forms of communication, WeChat and micro-blog have 
a very noticeable difference. As a media tool, Micro-blog is based primarily on interest relationships which are always weak and mostly one-way communication. Micro-blog's attention is given to the propagation speed and the content of public information. So, dissemination speed and breadth will be faster and wider in micro-blog than in WeChat. As a social tool, WeChat establishes relationships based on social relations. Its relationship quality is firm and the two-way relationship. WeChat's attention is paid to the exchange and interaction between private contents. So, its information dissemination speed is not fast, but its audience digestion rate is very high. Therefore, it is necessary to study the spread influence algorithm on WeChat subscription's topics. At present, the impact analysis algorithm of micro-blog mainly refers to the Google Page Rank (Page, 1998) algorithm and improved algorithm (Lamberti, Sanna \& Demartini, 2008; Li, Chen, Wang \& Zhang 2011). HITS (Kleinberg, 1999) improved algorithm (Asano, Yu \& Nishizeki, 2007; Liu \& Lin, 2007) draws on the idea of Page Rank algorithm. Tunke lang constructs a link directed graph and proposes the Twitter Rank algorithm (Weng, Lim, Jiang \& He, 2010). The algorithm is mainly to measure the impact of users on a topic. The main idea is that given a topic which the user's influence is meant by the influence of all his fans (Yang, Kefei, Shiren, Yan, Ding, \& Yang, 2012). However, the algorithm only considers the interaction between users with similar topics and is also not applicable to the WeChat subscription's article dissemination characteristics. Because the WeChat subscription's information dissemination characteristics have the driving frequency flexibility and the posting time is not a fixed characteristic, it makes subscription's graphics and text push more 'casual' but not 'arbitrary'. Users have their own reading habits, so it is necessary to analyze the relationship between the WeChat subscription posting time and the subscription influence. In WeChat research field, the document (Liao, Shi, Chi \& Cheng, 2017) made a detailed review of literature on blended learning and WeChat. Other researchers (Qiu, Li, Chen, Chen, Chen \& Chen, 2017) analyze the daily usage logs from WeChat group messaging platform with the goal of understanding the processes by which social messaging groups come together, grow new members, and evolve over time. The document (Mao, Liu, Zhang $\& \mathrm{Ma}, 2014)$ analyzed the user's influence by using the time and quantity of micro-blog released by micro-blog users, but this method did not introduce the actual topic to analyze the impact of users on a social problem. Therefore, this paper proposes a WeChat subscription topic influence model based on WeChat subscription's own specific communication ability, time characteristics and communication capacity numerical characteristics. So that it can truly and effectively reflect the WeChat subscription articles' topic spread of influence.

In this paper, we describe a model for analyzing the influence of the WeChat subscription articles' topic communication influence and focus on three central questions. We have collected articles from Tencent -WeChat subscription data and the reviews of the article's data to analyze.

1. Does the time of dispatch of WeChat subscription have certain regularity? Will the time of posting affect the impact of the WeChat subscription?

2. How to measure the similarity of topics between two subscriptions and how to establish communication connections between the WeChat subscriptions?

3. How to reasonably set the influence index of WeChat subscription's article?

In next section, we consider each of our three main questions in detail.

\section{THE MODEL}

\subsection{WeChat Subscription's Time-Parameter Model}

There is a close relationship between the WeChat subscription's posting time and its influence. Therefore, the article posting time is a key variable affecting the effectiveness of WeChat subscription's information dissemination. We can use the article's status like the amount of reading, the amount of 
the favorite and the number of comments in each time period to analyze the WeChat subscription user's reading habits. We census these data within each time period to establish WeChat subscription's articles influence time parameter model.

The number of readings, favorites, and reviews of WeChat subscription's articles in each time period are shown in Figure 1.

Figure 1 shows the amount of reading, praise and comments on the overall distribution of various periods of time. Using time distribution characteristics of WeChat subscription, we can calculate the WeChat subscription's propagation characteristics at different time periods. More than $40 \%$ of the WeChat subscription's article dissemination parameters appear in the 16:00-20:00 period, indicating that users are most likely to read at this time.

The percentage of the WeChat subscription's article during each time period is shown in Figure 2.

Figure 2 shows the relation between the number of subscription's posting and the time of dispatch, from which we can see the delivery time is between 13:00 and 21:00. Because of the limitation of reading ability and reading time, if subscription's delivery time is in peak delivery time, it will restrict the subscription's influence ability.

Figure 1 and Figure 2 show that the posting situation at different time periods is linked to the scope of the crowd affected by the subscription's article. Therefore, we have chosen to establish the relationship model between the subscription's influence power and the article push time. We expect the subscription of posting periods as the input to the model and predict the maximum influence of subscription's articles at this time. We can forecast the best time when the subscription can affect most people.

Figure 1. The Time distribution of WeChat subscription's article reading, point praise, comments

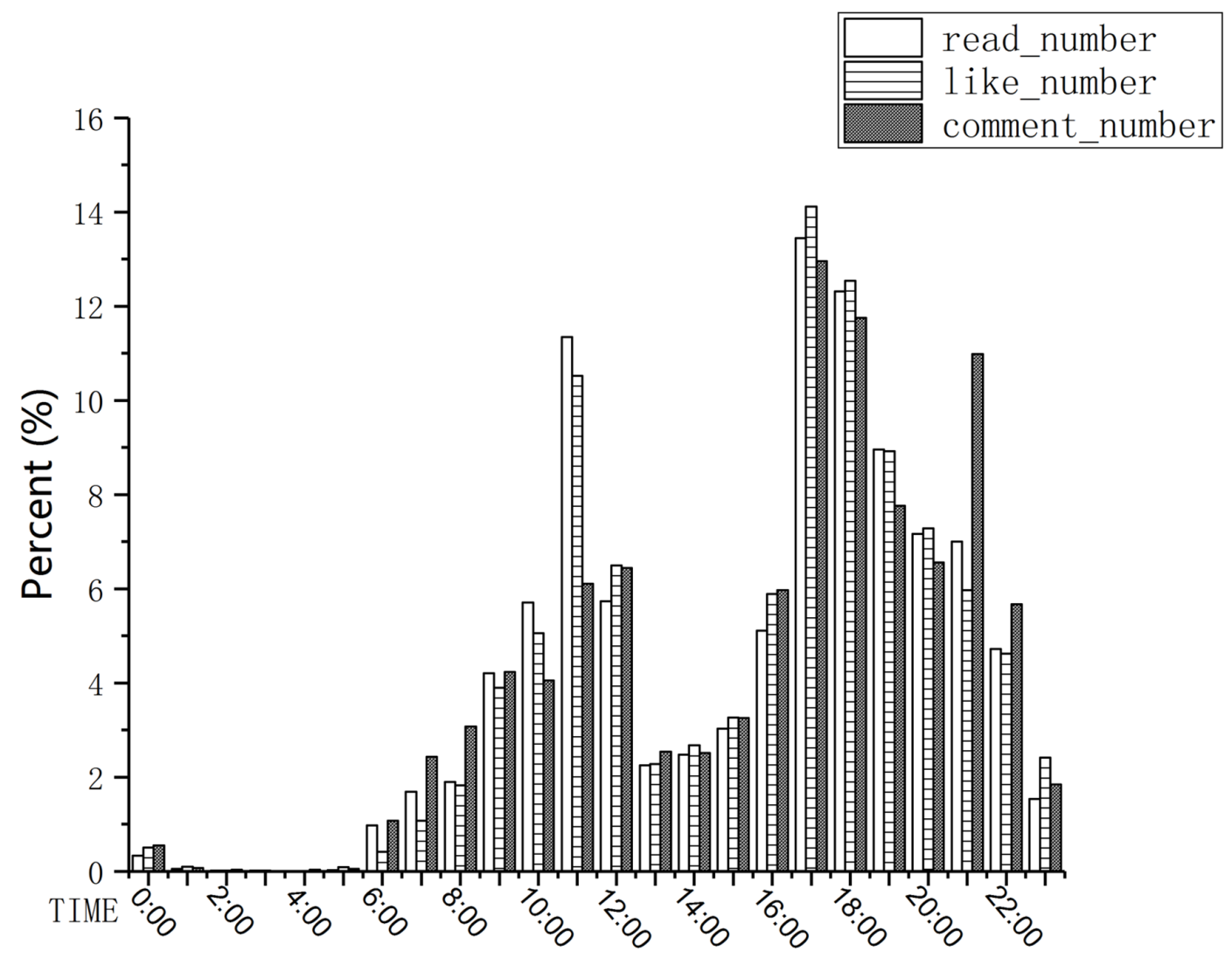




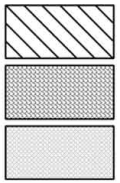

$7: 00-9: 00$
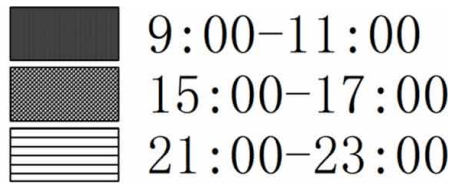

\section{9. $1 \%$}

$13: 00-15: 00$

$19: 00-21: 00$

$21: 00-23: 00$

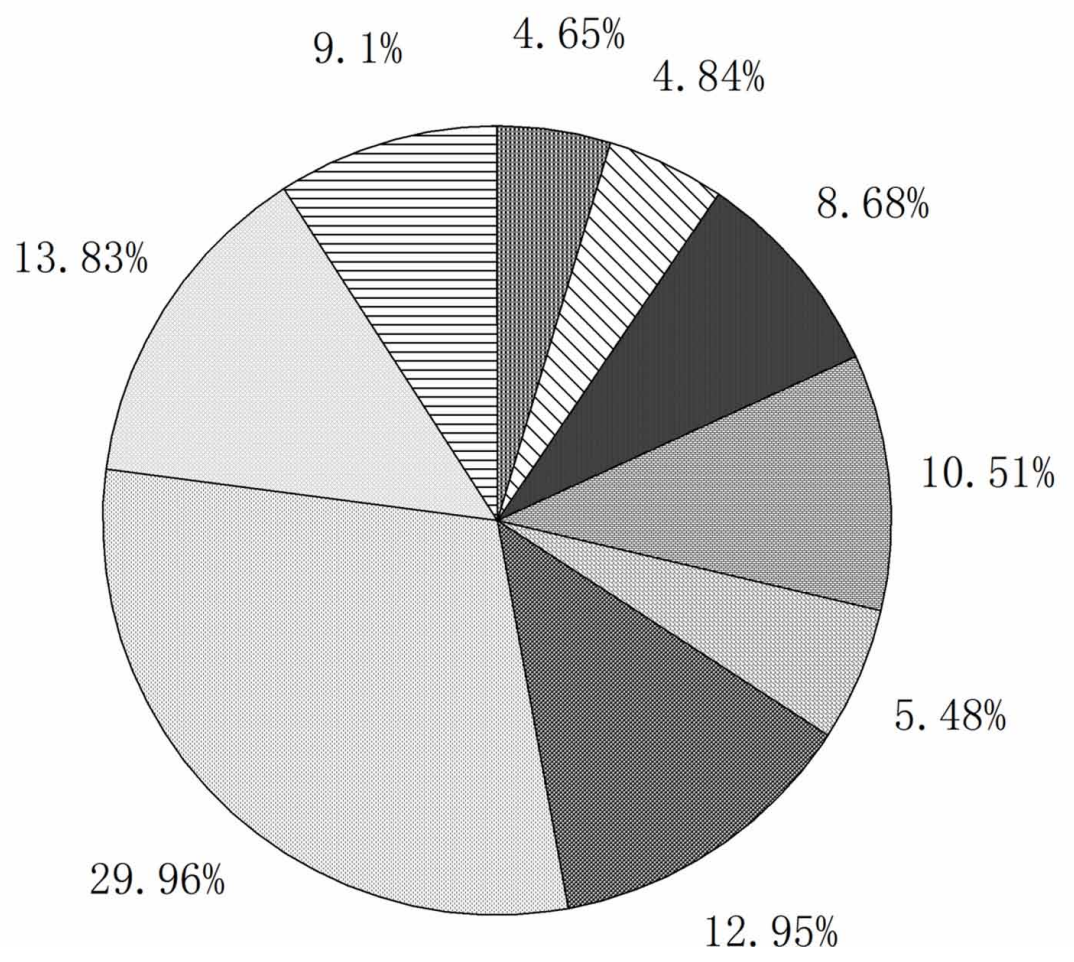

We assess the amount of reading, the amount of praise, the distribution of comments, and the time parameters of the peak time. Then we extract the numerical parameters of WeChat subscription in each period and the features of these numbers. Finally, we can get the relationships between propagation ability and time distribution parameters of the subscription. The characteristic parameters for input training are the parameters of maximum value of subscription's readings, praise, comments and the frequency of subscription's article posting time in a period of time, the average reading number, average praise and average comment number in each period of time. We set up the time parameter model of subscription's article influence.

The time parameter model uses curves to make the parameterization more flexible (Rowe, 2014). We suppose subscription u releases $n_{u}$ article. Specify $k_{u}$ time points $\left\{\mathrm{t}_{1}^{u}, \& \&, \mathrm{t}_{k_{u}}^{u}\right\}$. These time points divide the posting time of the subscription evenly and act as a function under the kernel control.

$\mathbf{b}_{u}(t)=\frac{\sum_{l=1}^{k_{u}} e^{-\sigma\left|t-t_{l}^{u}\right|} b_{t_{l}}^{u}}{\sum_{l=1}^{k_{u}} e^{-\sigma\left|t-t_{l}^{u}\right|}}$ 
International Journal of Digital Crime and Forensics

Volume $11 \cdot$ Issue $4 \cdot$ October-December 2019

The parameter $b_{t_{l}}^{u}$ is related to the control point (called the kernel). Automatic learning is obtained by training data in the article $n_{u}$ published by the subscription $\mathrm{u}$. In this way, the propagation parameter model of subscription is composed of a weighted combination of these time parameters. The number of control points $k_{u}$ can be used to reconcile algorithm flexibility and computational efficiency. Here, $k_{u}=24$ divides the day by an hour. The constant determines the smoothness of the curve, and cross validation sets $\alpha$ to 0.3 . Figure 3 shows the results for this analysis.

As shown in Figure 3, the average probability of the subscription can reach the maximum spread influence when it is released within each time period. When the subscription sends an article at 11:00, 18:00, and 19:00, it will have a high probability of affecting more people. The line shows the influence of a rising trend between 8:00 and 11:00. After 11:00, there is a clear descending trend. The period from 14:00 to 19:00, the subscription's influence will have the second upward trend. The period from 19:00 to 23:00, has a downward trend. From 23:00 to 5:00 the next day, the influence of the subscription basically presents a steady trend and the impact of the largest average probability of almost zero. At the same time, this trend of change accords with the subscription of users' reading and work habits.

Figure 3. The distribution of WeChat subscription influence at different time periods

--Percentage of communication influence

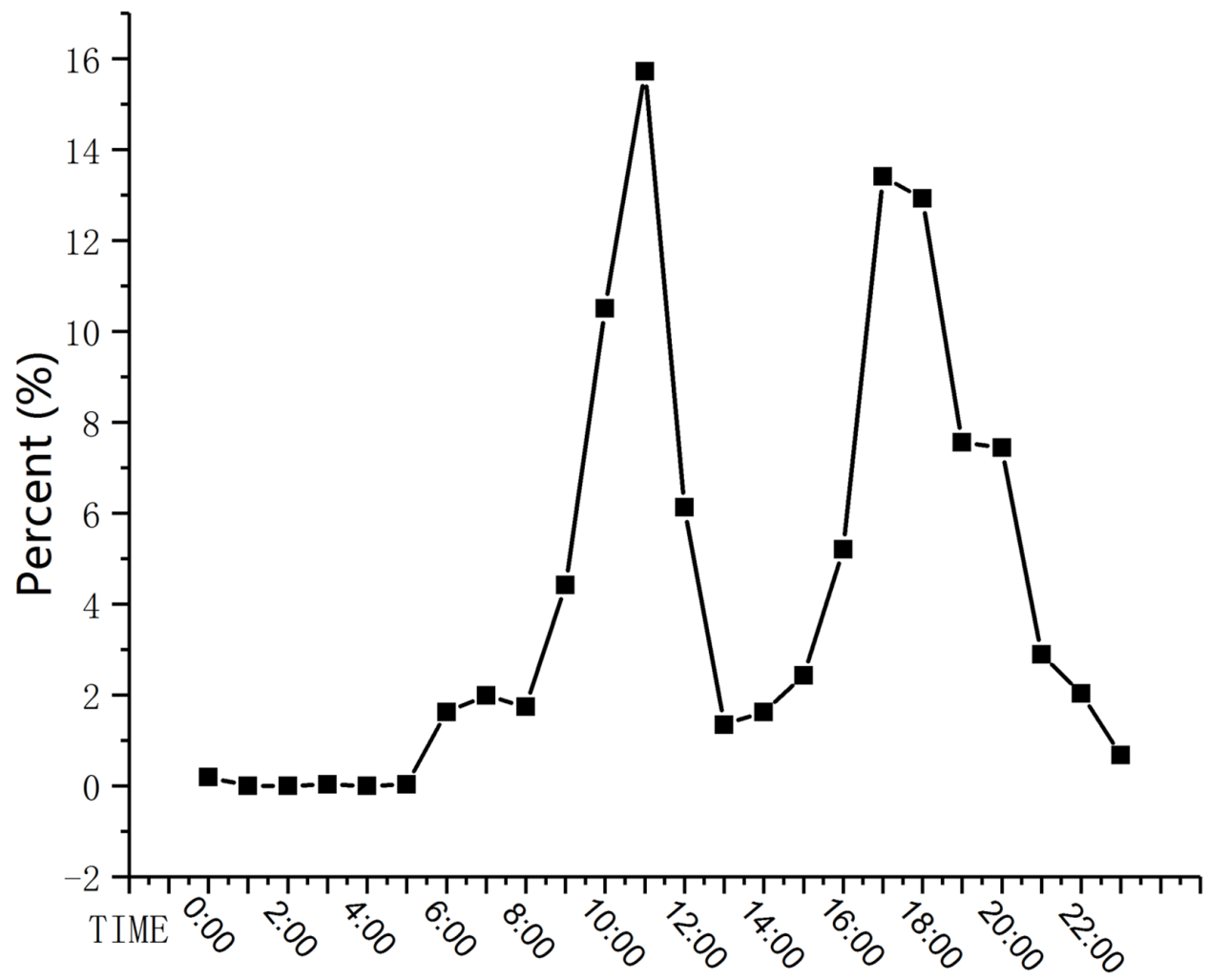

134 


\subsection{WeChat Subscription's Topic Clustering}

WeChat subscriptions publish mostly concise texts. The brief texts have short length and relatively little information and so it has a worrying feature sparseness problem. If we use traditional text analysis method, it will occur the problem of difficulty in the similarity calculation and instability of clustering results in text clustering analysis (CHENG, HOU, TENG, 2015; Qiu, Wang, Shao, Guo, Software \& University, 2014). We use HowNet to extend semantic information of short texts and compute the influence similarity where we introduce the characteristics of the propagation parameter of the subscription, such as the amount of reading, the praise of the article and so on. Finally, the results are linearly weighted.

In order to reduce the complexity of the algorithm and enhance the effectiveness of the algorithm (Huang, Chen, Yan \& Luo, 2005), we should cut WeChat subscription's article word segmentation, and eliminate the useless function words, stop words, error words and spam information to clean the text information. In this paper, Chinese lexical analysis (ICTCLAS) developed by Chinese Academy of Sciences is used for word segmentation.

HowNet is a knowledge system that describes the relationship between conceptual relationships and conceptual attributes (You, Yan, Sun \& Liu, 2013). On two Chinese words $W_{1}, W_{2}$, if they have n senses like $F_{11}, F_{12}, \& \& F_{1 \mathrm{n}}$, and have m meanings such as $F_{21}, F_{22}, \& \& F_{2 m}$. The similarity between $W_{1}$ and $W_{2}$ is the maximum of the similarity of each semantics.

$$
C V\left(W_{1,} W_{2}\right)=\max _{i=1,2, \ldots, n} \underset{j=1,2, \ldots, m}{C V}\left(F_{1 \mathrm{i}}, F_{2 \mathrm{j}}\right)
$$

All the semantic elements form a tree hierarchy based on the upper and lower relations. By using semantic distance to compute the acquaintance degree, the semantic distance between two semantic bases and the path distance in the tree structure is D. The two semantic elements are defined as.

$$
C V\left(R_{1}, R_{2}\right)=\frac{\theta}{d+\theta}
$$

Formula: where d means the path length of $R_{1}$ and $R_{2}$ in tree structure, $\theta$ means the adjustable parameter. A substantive description includes basic original description, other basic original description, relational semantic description, and relational symbol description. Therefore, the overall similarity of the two semantic concepts is calculated as follows.

$$
C V\left(W_{1}, W_{2}\right)=\sum_{i=1}^{4} \phi_{i} \prod_{j=1}^{4} C V\left(W_{1}, W_{2}\right)
$$

Where $\phi$ means adjustable parameters, $\phi_{1}+\phi_{2}+\phi_{3}+\phi_{4}=1, \phi_{1} \geq \phi_{2} \geq \phi_{3} \geq \phi_{4}$.

Let $\mathrm{u}$ denote the WeChat subscription's article numeric data. $r_{u}$ is the amount of reading of the article, $f_{u}$ is the amount of praise of the article, $c_{u}$ is the amount of comment of the article, and $\mathrm{b}_{u}(t)$ is the time bias parameter of the article. These features are represented as a vector $r=\left(r_{u}, f_{u}, c_{u}, \mathrm{~b}_{u}(t)\right)$. Pearson similarity is used to measure the similarity of spreading ability. Let $r_{u i}$ denote the i numerical characteristics of WeChat $\mathrm{u}$ article. $\bar{r}_{u}$ is the average of all the WeChat subscription $\mathrm{u}$ the numerical 
data in a period of time. Therefore, the similarity between the WeChat subscription $\mathrm{u}$ articles and articles for WeChat subscription $\mathrm{v}$ is calculated as follows.

$$
P C(u, v)=\frac{\sum_{i \in I_{u v}}\left(r_{u i}-\overline{r_{u}}\right)\left(r_{v i}-\overline{r_{v}}\right)}{\sqrt{\sum_{i \in I_{u v}}\left(r_{u i}-\bar{r}\right)^{2} \sum_{i \in I_{u v}}\left(r_{v i}-\overline{r_{v}}\right)^{2}}}
$$

\subsection{WeChat Subscription's Topic Propagation Similarity}

HowNet calculates the subscription's article similarity and PC algorithm calculates the influence similarity combines linearly. We get the similarity results. We use $\alpha$ to denote subscription's article. Then, the similarity between $\alpha_{1}$ and $\alpha_{2}$, is calculated as follows.

$$
\operatorname{sim}\left(\alpha_{1}, \alpha_{2}\right)=\xi \times C V\left(\alpha_{1}, \alpha_{2}\right)+(1-\xi) \times P C\left(\alpha_{1}, \alpha_{2}\right)
$$

$\xi(0 \leq \xi \leq 1)$ is the linear parameters. Between the two similarities, which has greater impact on the results cannot be identified in advance. The algorithm of similarity combination is calculated as Table 1.

\subsection{WeChat Subscription's Quality-Parameter}

Based on the BRR algorithm and the characteristics of the WeChat subscription, several parameter indexes (Cha, Haddadi, Benevenuto \& Gummadi, 2010) for WeChat subscription Quality evaluation are proposed. As shown in Table 2 below.

In Table 2, the quality factor of the publication is expressed as the average quantity of each posting article being read and praised. When WeChat subscription's articles cause the reader's resonance, the user will participate in interactions to express their views and opinions. Reading praise ratio will show the influence degree of the specific reading population to a certain extent and the influence

Table 1. The algorithm of similarity combination

\begin{tabular}{|l|l|}
\hline \multicolumn{2}{|l|}{ Algorithm 1} \\
\hline 1: & Input: Text set $\alpha=\left\{\alpha_{1}, \alpha_{2}, \ldots, \alpha_{n}\right\}$ \\
\hline 2: & $\begin{array}{l}\text { Using ICTCLAS segmentation system to segment and delete the text set } \mathrm{D}, \text { and the feature word set is filtered out } \\
\text { from the segmentation words } \mathrm{T} .\end{array}$ \\
\hline 3: & $\begin{array}{l}\text { Calculating the semantic similarity using the HowNet dictionary, and calculating the text similarity between the } \\
C V\left(\alpha_{i}, \alpha_{j}\right) .\end{array}$ \\
\hline 4: & $\begin{array}{l}\text { According to the numerical characteristics of WeChat's articles, the similarity } P C\left(\alpha_{i}, \alpha_{j}\right) \text { is calculated by } \\
\text { using the PC algorithm. }\end{array}$ \\
\hline 5: & $\begin{array}{l}\text { Combining the linear combination parameter theta, the similarity } \operatorname{sim}\left(\alpha_{i}, \alpha_{j}\right) \text { between the texts is calculated, } \\
\text { and then the final similarity result set } \mathrm{S} \text { is obtained. }\end{array}$ \\
\hline
\end{tabular}


Table 2. WeChat subscription quality evaluation index

\begin{tabular}{|l|l|}
\hline First level index & Two level index \\
\hline Article quality coefficient & Reading quantity \\
\cline { 2 - 2 } & Favorite quantity \\
\cline { 2 - 2 } & Comment quantity \\
\hline \multirow{2}{*}{ User activity } & Publish article quantity \\
\cline { 2 - 2 } & Number of uninterrupted articles \\
\hline
\end{tabular}

range of the amount of reading will play a key role in the propagation. User's reading ability in each period of time has a certain effect. The article quality coefficient indicates the function as follows.

$$
\begin{aligned}
& Q_{i}=\frac{\lambda_{R} \times R_{i}+\lambda_{L} \times L_{i}+\lambda_{C} \times C_{i}}{N_{i}} \\
& \lambda_{R}+\lambda_{L}+\lambda_{C}=1
\end{aligned}
$$

$Q_{i}$ is the WeChat article quality coefficient of the subscription i . $R_{i}$ is the amount of reading for the WeChat subscription i. $L_{i}$ is the amount of praise for the WeChat subscription i. $C_{i}$ is the number of comments for the WeChat subscription i. $N_{i}$ is the total number of sending articles for the WeChat subscription i. $\lambda_{R}$ is the weighted value of the number of reading, and $\lambda_{L}$ is the weight value of praise, and the $\lambda_{C}$ is the weight value of the comment quantity. For $\lambda_{C}$, the size can be adjusted according to the condition. If the WeChat subscription does not have a comment standard, $\lambda_{C}$ is 0 .

Another index is an indicator of active users of WeChat, which means that the subscription's posting is frequent and regular. The more articles the subscription sends, the more active the subscription is. $N_{i}$ is the total number of sending articles for the WeChat subscription i . $Y_{i}$ indicates the number of days in which subscription's articles are published continuously. These two parameters reflect how active the subscription is. $\alpha_{N}$ and $\beta_{Y}$ represent its weight. The size of the weights is determined by the maximum number of reading and the number of the highest praise. The function of subscription activity is expressed as follows.

$$
A_{1}=\frac{\alpha_{N} \times N_{i}}{T}+\beta_{Y} \times Y_{i}
$$

$A_{1}$ represents the activity of the WeChat subscription i. $N_{i}$ indicates the total number of sending articles for the WeChat subscription $\mathrm{i} . \mathrm{T}$ is the time parameter. The article quality factor and subscription activity can well reflect the subscription's influence in the community.

In the BRR algorithm, if the subscription has the higher the quality coefficient of the article and the greater the user activity, subscription will have the greater the impact of the community.

According to the above analysis, the formula of subscription quality is expressed as follows. 
$S_{i}$ is quality of the subscription i. $Q_{i}$ is determined by the posting of the subscription's article quality factor. $A_{1}$ is the subscription activity

\subsection{TIPN Model}

We analyze the user's comment data. The link between the WeChat subscriptions is established through the fan's comments in the same topic and the similarity between the subscriptions (Kempe, Kleinberg \& Éva, 2003). The WeChat subscription propagation influence relationship network diagram is $\mathrm{G}=(\mathrm{V}, \mathrm{E})$ which consists of the set of vertices $\mathrm{V}$ and the set of edges $\mathrm{E}$ (Kloumann, Ugander \& Kleinberg, 2017; Page, 1998). The formula for calculating the influence size of the WeChat subscription is as follows.

$\operatorname{TIPN}\left(u_{i}\right)=\frac{(1-\omega)}{N}+\frac{\omega \sum_{u_{i} \in M_{u i}} \operatorname{TIPN}\left(u_{i}\right)}{F\left(u_{i}\right)}$

$M_{u i}$ is set of subscriptions which have the same topic and have common fans. $u_{i}$ represents the subscription. $\mathrm{N}$ is the number of subscriptions which are in the same topic. The similarity of the topic is measured by WeChat subscription's Topic Propagation Similarity. $\omega$ represents damping factor, with a range of $0-1$. According to the formula above, the TIPN value which we can calculate is the final result when the iteration is steady.

The matrix is used to represent the connection between the subscriptions. The initial matrix is $A=\left(A_{i, j}\right) . A_{i, j}$ represents the probability that subscription information $\mathrm{j}$ can be transmitted from its fans to the subscription i. For $i, j \in G$. The related matrix of directed graph $G$ is set as follows:

$$
A_{i, j}=\frac{1}{F(j)}, \text { when }<j, i>\in E
$$

$$
A_{i, j}=0, \text { when }<j, i>\notin E
$$

$F(j)$ is $\mathrm{j}$ fans associated with the number of subscription which have the same topic.

$$
g m_{i, j}=\left(\omega \times A_{i, j}+\frac{(1-\omega)}{N}\right) \times S_{i}, \text { when }<j, i>\in E
$$

Suppose that $\mathrm{n}$ is the number of nodes in graph G. GM is a matrix made up of $g m_{i, j}$. Therefore $T I P N_{n}=G M \times T I P N_{n-1}$. Update the rank vectors are updated by step to complete the iteration until the $T I P N_{n}=T I P N_{n-1}$ is obtained. 


\section{EXPERIMENTS}

We have conducted extensive experiments to study the proposed approach over a system equipped with Intel i7 $2.60 \mathrm{GHz} \mathrm{CPU}$, and $8 \mathrm{~GB}$ memory. Our datasets are random sample of 1000 subscriptions which are selected from the WeChat subscription and include 310649 articles.

\subsection{Datasets}

WeChat on-line social network is one of the largest on-line social network in China, which was claimed to have more than 653 million monthly active users by September 30, 2015. We collected the history data of WeChat which consists of 1000 subscriptions. The selected subscription data are collected from May 2016 to May 2017. WeChat subscriptions of 310649 articles are selected based on numerical data. The result of data analysis is presented in Table 3 and Table 4.

Table 3 shows the changes of the subscription's influence in different time periods for distinct propagation parameters.

Table 4 shows the WeChat subscription post status from 0:00 to 23:00. Table 5 demonstrates some data of fans that follow in some food related topics and the data are sorted by the number of associated subscription.

The first column of Table 5 is the subscription's name, and the second is the subscription's comment data. In the three columns of the comment data, the first column is the number of subscription which is associated with the same fans for a period of time. The second column is the nickname of the subscription's fans. And the third column is the number of comments of the fan on the subscription during this time. By analyzing the number of the fans' comments on the subscriptions' articles, the liveness and the degree of closeness relevance between subscriptions can be reflected. The number of fans who are concerned with the same topic and their number of comments in different WeChat subscriptions can reflect the superposition area between the connected relationship of fans and the dissemination of information. We establish a communication network using the subscription's comments of the fans.

\subsection{Performance Metrics}

We conduct the experiments in four different real social networks. In this work, our goal is to evaluate the relationship between the WeChat subscription's posting time and its influence. We have used data samples extracted from WeChat. Furthermore, we practically evaluate TIPN model and find the key communication nodes of same topic.

Figure 4 shows the characteristics of the propagation of the articles with different posting times. The amount of reading, praise, and comment in each period of time is consistent. The optimum propagation time is centered at 11:00,17:00 and 18:00. For comment data, there is only an obvious upward trend in the 20:00-21:00 period. We can speculate that the ability of the subscription's fans to read and interact with them is better to some extent at this time.

We analyze the relationship between the issuing time and the communication ability of the subscription. Users' daily reading distribution on articles is influenced by the user's reading habits and user's free time of the day. The distribution of the reading number in one day is shown in Figure 5 which X label is posting time and Y label is Propagation ability. The starting point is 00:00:00 in the morning and it is signed as 0 seconds on the abscissa axis. The ending point is 86399 second on the abscissa axis. The ordinate indicates the number of people affected during this time period. The more overlapping circles in the figure, the more intensive the subscription posting. The size of the circle indicates the probability that the number of people affected at this time will reach the number shown on the ordinate. It can also be expressed as a larger circle indicates that the big probability can affect more people when subscriptions release articles in this period. We analyze that the least reading quantity of the article appears during the period of time from 8000 to 20000 seconds and article published most intensively during the period of time from 20000 to 84000 seconds. We analyze 
Table 3. Subscriptions' article dissemination of numerical data

\begin{tabular}{|c|c|c|c|c|c|c|c|c|c|}
\hline \multirow{2}{*}{$\begin{array}{c}\text { Data } \\
\text { Time }\end{array}$} & \multicolumn{3}{|c|}{ Read_Number } & \multicolumn{3}{|c|}{ Like_Number } & \multicolumn{3}{|c|}{ Comment_number } \\
\hline & Mean & $\begin{array}{c}\text { Percent } \\
(\%)\end{array}$ & total & Mean & $\begin{array}{c}\text { Percent } \\
(\%)\end{array}$ & total & Mean & $\begin{array}{c}\text { Percent } \\
(\%)\end{array}$ & Total \\
\hline 0:00 & 1979.48 & 0.33 & 5063501 & 19.67 & 0.51 & 50306 & 1.39 & 0.55 & 3548 \\
\hline $1: 00$ & 2559.93 & 0.05 & 791019 & 30.29 & 0.09 & 9360 & 1.51 & 0.07 & 467 \\
\hline $2: 00$ & 3202.00 & 0.01 & 185716 & 26.40 & 0.02 & 1531 & 4.19 & 0.04 & 243 \\
\hline 3:00 & 4362.98 & 0.01 & 226875 & 35.98 & 0.02 & 1871 & 1.00 & 0.01 & 52 \\
\hline 4:00 & 1317.35 & 0.01 & 137004 & 8.00 & 0.01 & 832 & 2.18 & 0.04 & 227 \\
\hline 5:00 & 498.70 & 0.03 & 440350 & 9.81 & 0.09 & 8662 & 0.39 & 0.05 & 341 \\
\hline $6: 00$ & 4979.09 & 0.98 & 15021917 & 13.60 & 0.41 & 41017 & 2.29 & 1.07 & 6923 \\
\hline $7: 00$ & 4862.20 & 1.69 & 25900956 & 20.06 & 1.08 & 106846 & 2.94 & 2.43 & 15679 \\
\hline 8:00 & 3008.27 & 1.90 & 29174155 & 18.73 & 1.83 & 181643 & 2.05 & 3.07 & 19844 \\
\hline 9:00 & 5093.23 & 4.21 & 64607622 & 30.51 & 3.90 & 386999 & 2.16 & 4.24 & 27353 \\
\hline 10:00 & 6136.12 & 5.70 & 87611588 & 35.15 & 5.06 & 501816 & 1.83 & 4.05 & 26179 \\
\hline 11:00 & 8221.43 & 11.35 & 174286173 & 49.27 & 10.52 & 1044490 & 1.86 & 6.11 & 39425 \\
\hline $12: 00$ & 7683.08 & 5.73 & 88032784 & 56.31 & 6.50 & 645176 & 3.63 & 6.44 & 41614 \\
\hline $13: 00$ & 4924.61 & 2.25 & 34615074 & 32.17 & 2.28 & 226118 & 2.34 & 2.54 & 16427 \\
\hline $14: 00$ & 3816.21 & 2.48 & 38120156 & 26.56 & 2.67 & 265324 & 1.63 & 2.52 & 16259 \\
\hline $15: 00$ & 3236.31 & 3.03 & 46479948 & 22.56 & 3.26 & 324037 & 1.46 & 3.26 & 21036 \\
\hline $16: 00$ & 3035.01 & 5.11 & 78461187 & 22.60 & 5.89 & 584364 & 1.49 & 5.97 & 38551 \\
\hline 17:00 & 3935.30 & 13.45 & 206591564 & 26.69 & 14.12 & 1401064 & 1.59 & 12.96 & 83689 \\
\hline 18:00 & 4659.67 & 12.31 & 189126741 & 30.68 & 12.54 & 1245083 & 1.87 & 11.75 & 75899 \\
\hline 19:00 & 5802.72 & 8.95 & 137553473 & 37.34 & 8.92 & 885073 & 2.11 & 7.76 & 50135 \\
\hline 20:00 & 5713.02 & 7.16 & 110021352 & 37.55 & 7.29 & 723186 & 2.20 & 6.56 & 42342 \\
\hline 21:00 & 6318.27 & 7.00 & 107492803 & 34.82 & 5.97 & 592451 & 4.17 & 10.99 & 70952 \\
\hline $22: 00$ & 6446.56 & 4.72 & 72498017 & 40.79 & 4.62 & 458756 & 3.26 & 5.67 & 36628 \\
\hline 23:00 & 3155.53 & 1.54 & 23615998 & 32.04 & 2.42 & 239815 & 1.59 & 1.85 & 11926 \\
\hline
\end{tabular}

that the propagation conditions of the article published in the 22000 seconds to 45000 seconds and 58000 seconds to 72000 seconds are better.

The subscription's comments are linked by enthusiastic fans at the same time. The comments of fans on the subscription's article and different subscriptions that have the same fans which pay attention to the same topic are used to establish a network (Li, Chen, Wang \& Zhang, 2011) .

As shown in Figure 6: $\mathrm{G}=(\mathrm{V}, \mathrm{E}), \mathrm{V}$ is the set of vertices of the graph which is made up of different subscriptions' articles. The collection of edges $\mathrm{E}$ is composed of fans associated with the subscription. The number of edges between vertices is determined by the number of common fans between the subscriptions.

Figure 6 shows the relationship among the subscriptions network connected by comments between fans, which is not obvious ways to spread the information and key points in the graph. If we just use the relationship between fans, the network will not well reflect the spread of relationships between the subscriptions and the connection between the subscriptions very well. 
Table 4. Subscription delivery time data

\begin{tabular}{|l|l|l|l|l|l|}
\hline \multicolumn{1}{|c|}{ Data } & \multicolumn{2}{c|}{ Article_Number } & \multicolumn{1}{c|}{ Data } & \multicolumn{2}{c|}{ Article_Number } \\
\hline \multicolumn{1}{|c|}{ Time } & \multicolumn{1}{|c|}{ Total } & \multicolumn{1}{c|}{ Percent $(\%)$} & \multicolumn{1}{c|}{ Time } & \multicolumn{1}{c|}{ total } & \multicolumn{1}{c|}{ Percent (\%) } \\
\hline $0: 00$ & 2558 & 0.82 & $12: 00$ & 11458 & 3.69 \\
\hline $1: 00$ & 309 & 0.10 & $13: 00$ & 7029 & 2.26 \\
\hline $2: 00$ & 58 & 0.02 & $14: 00$ & 9989 & 3.22 \\
\hline $3: 00$ & 52 & 0.02 & $15: 00$ & 14362 & 4.62 \\
\hline $4: 00$ & 104 & 0.03 & $16: 00$ & 25852 & 8.32 \\
\hline $5: 00$ & 883 & 0.28 & $17: 00$ & 52497 & 16.90 \\
\hline $6: 00$ & 3017 & 0.97 & $18: 00$ & 40588 & 13.07 \\
\hline $7: 00$ & 5327 & 1.71 & $19: 00$ & 23705 & 7.63 \\
\hline $8: 00$ & 9698 & 3.12 & $20: 00$ & 19258 & 6.20 \\
\hline $9: 00$ & 12685 & 4.08 & $21: 00$ & 17013 & 5.48 \\
\hline $10: 00$ & 14278 & 4.60 & $22: 00$ & 11246 & 3.62 \\
\hline $11: 00$ & 21199 & 6.82 & $23: 00$ & 7484 & 2.41 \\
\hline
\end{tabular}

We analyze the data of some subscription's articles and the TIPN value of the subscription nodes (which have been translated into 100 percent) is shown in Table 6.

The first column of Table 6 is the registration of the WeChat subscription ID. The second part is the analysis of these subscriptions. The first column of the second part is the maximum range of posting time for subscription between January 1, 2016 and December 12, 2016. The second column is the proportion of original articles in the subscription. The third column is the ratio of the repost articles of the subscription. The fourth column is the percentage between image and data in the articles of the subscription. The last column is the TPIN value, which reflects the strength of the subscription's ability in similar topics. If the TPIN value is high, the subscription's influence on the dissemination in similar topics is strong. If a subscription has smaller TPIN value, it shows that the communication capacity of the subscription is weaker.

We cluster WeChat subscription's articles topics and extract the features of the subscription' fans relationship. Then, we use them as the input of TIPN algorithm to get the influence of the WeChat subscriptions on the same topic in WeChat. The subscription node of the same topic is posted on the network, in which the key nodes in the propagation process are dependent on the topic community. As shown in Figure 7.

Figure 7 shows the subscription relations network of influence. Depending on the calculation result of TIPN algorithm, the relation matrix of node influence is obtained. We will find the key node which has high TIPN value and appears in the center of the cluster. When the WeChat subscription information is transmitted, we argue that the node, which plays a key role in the communication process, has the greatest impact. The TIPN algorithm adds the time bias parameter of subscription, so we can discuss the difference of the communication influence in different posting time. The left of Figure 7 is an individual topic propagation network of a subscription in the peak period (16:0020:00). The other side of Figure 7 is communication network of the same single topic during the 12:00-16:00 period. It shows that in discrete time dimensions, the impact of topic propagation is obviously distinct, and the number of key nodes is also different.

Figure 8 displays the distinct topics of subscription's communication network. According to diverse topic nodes TIPN values, the relation matrix of propagation influence is obtained. We will find the key communication nodes of discrete topics. These nodes which make up a community 
Table 5. WeChat subscription's fans associated data

\begin{tabular}{|c|c|c|c|}
\hline \multirow[t]{2}{*}{ subscription } & \multicolumn{3}{|c|}{ Comment data } \\
\hline & Associated subscription & nickname & Fans comment quantity \\
\hline Chinese Zhongche & 8 & $\begin{array}{l}\text { The strongest surface of the } \\
\text { earth }\end{array}$ & 546 \\
\hline Macao doulao & 5 & Fashion City & 5 \\
\hline Pizza hut & 4 & ChengXi & 33 \\
\hline PapaJohns & 4 & JUST & 2 \\
\hline $\begin{array}{l}\text { Kentucky Fried } \\
\text { Chicken }\end{array}$ & 4 & ?XiaoMing ${ }^{2016}$ & 6 \\
\hline Huang Jihuang & 4 & ?Shu Yu? & 7 \\
\hline Dianrong Network & 4 & home and everything & 301 \\
\hline Dicos & 3 & Mystery man & 2 \\
\hline HappyLemon & 3 & HitomiLiu & 8 \\
\hline Starbucks China & 3 & Queen? & 10 \\
\hline hot-pot Haidilao. & 3 & Linmax & 14 \\
\hline MacDonald & 3 & Westwood and Muskmelon & 32 \\
\hline Yoshinoya & 2 & Fang Niuer & 3 \\
\hline $\mathrm{CoCo}$ & 2 & I'll...?? & 3 \\
\hline BreadTalk & 2 & MaoMao & 3 \\
\hline COSTACOFFEE & 2 & Invisible Man & 4 \\
\hline Huang Taiji Club & 2 & $\mathrm{P} \& \mathrm{C}$ & 5 \\
\hline BaiFu catering & 2 & With love as a radius & 6 \\
\hline Beijing Feast & 2 & $\begin{array}{l}\text { Li Zhiqiang-Shuanghe- } \\
\text { catering }\end{array}$ & 6 \\
\hline $\begin{array}{l}\text { Oriental Dumpling } \\
\text { King }\end{array}$ & 2 & Spikey & 37 \\
\hline
\end{tabular}

have higher impact in the same topic. These communities play a key role in the dissemination of this topic. The TIPN algorithm can also show the impact of the topic propagation in different temporal dimensions. The left half of Figure 8 is the network of influence relationships between 1000 users of different topics within a week. The right half of Figure 8 is a composite network of 9 topics in one day that show the relationships between different topics. We can analyze the degree of association between these nine topics that show the amount of forwarding and inclusion of entertainment topics is the largest.

\section{CONCLUSION}

This paper presents a novel model for WeChat subscription influence. This model takes the WeChat subscriptions' posting time, topics of subscription and the relationship between the subscriptions connected by comments between fans into consideration to TIPN model. In the actual evaluation, we use the WeChat real data set for simulation. If the subscription has a high value of TPIN, it has more comment fans with other subscriptions and its number of reading and praises is also high. We 


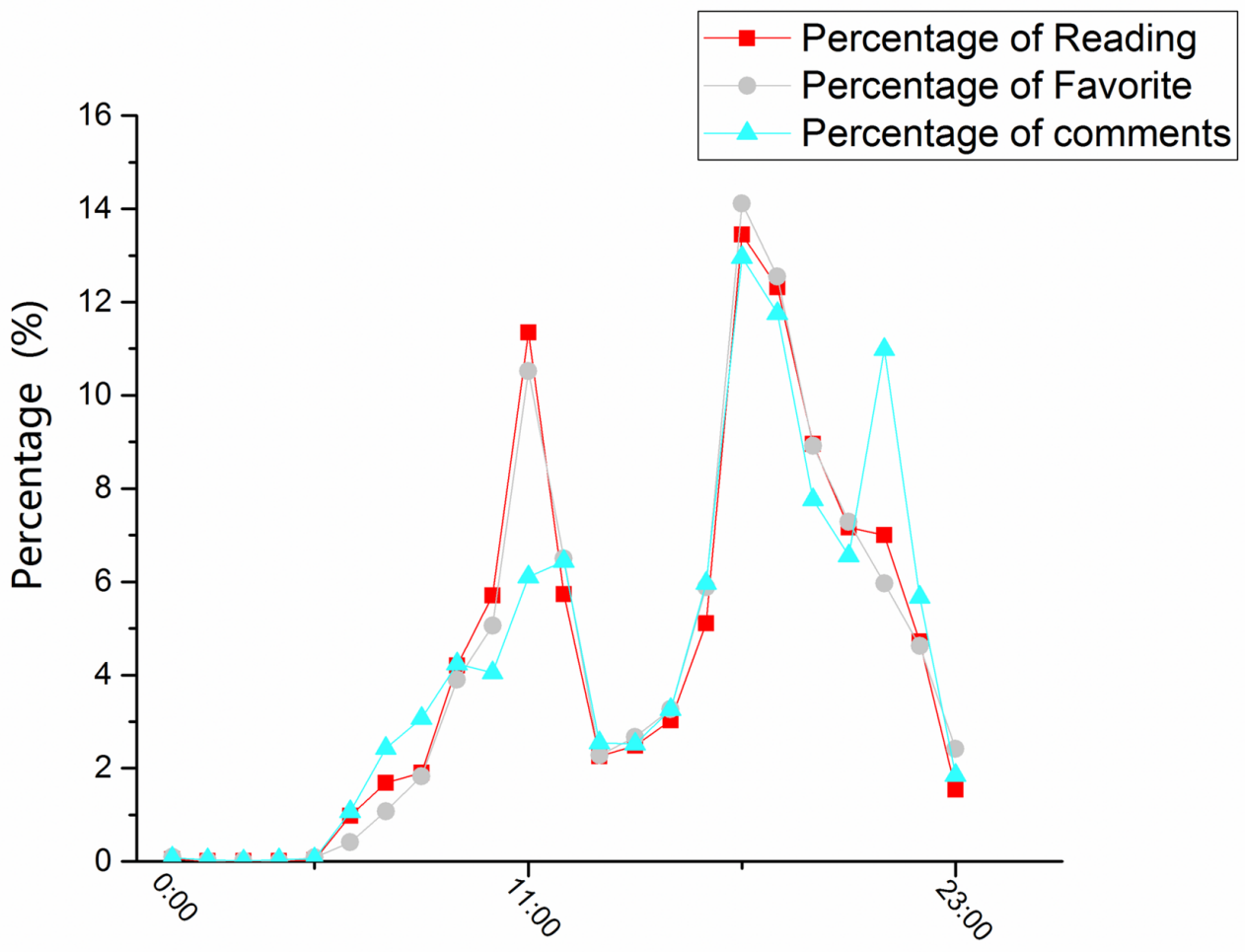

further evaluated the TPIN model, it is also able to handle the subscription node of the same topic and diverse topic where it releases in different temporal dimensions. Furthermore, we practically evaluate the WeChat subscription and selected key subscriptions in the communication process. Experimental results demonstrated the effectiveness of our proposed model. Currently, we only consider the relationship between the subscriptions connected by comments between fans in the proposed model. In the future, we will extend our work to the WeChat subscription real readers and users. 
International Journal of Digital Crime and Forensics

Volume 11 • Issue 4 • October-December 2019

Figure 5 . The relationship between propagation ability and posting time

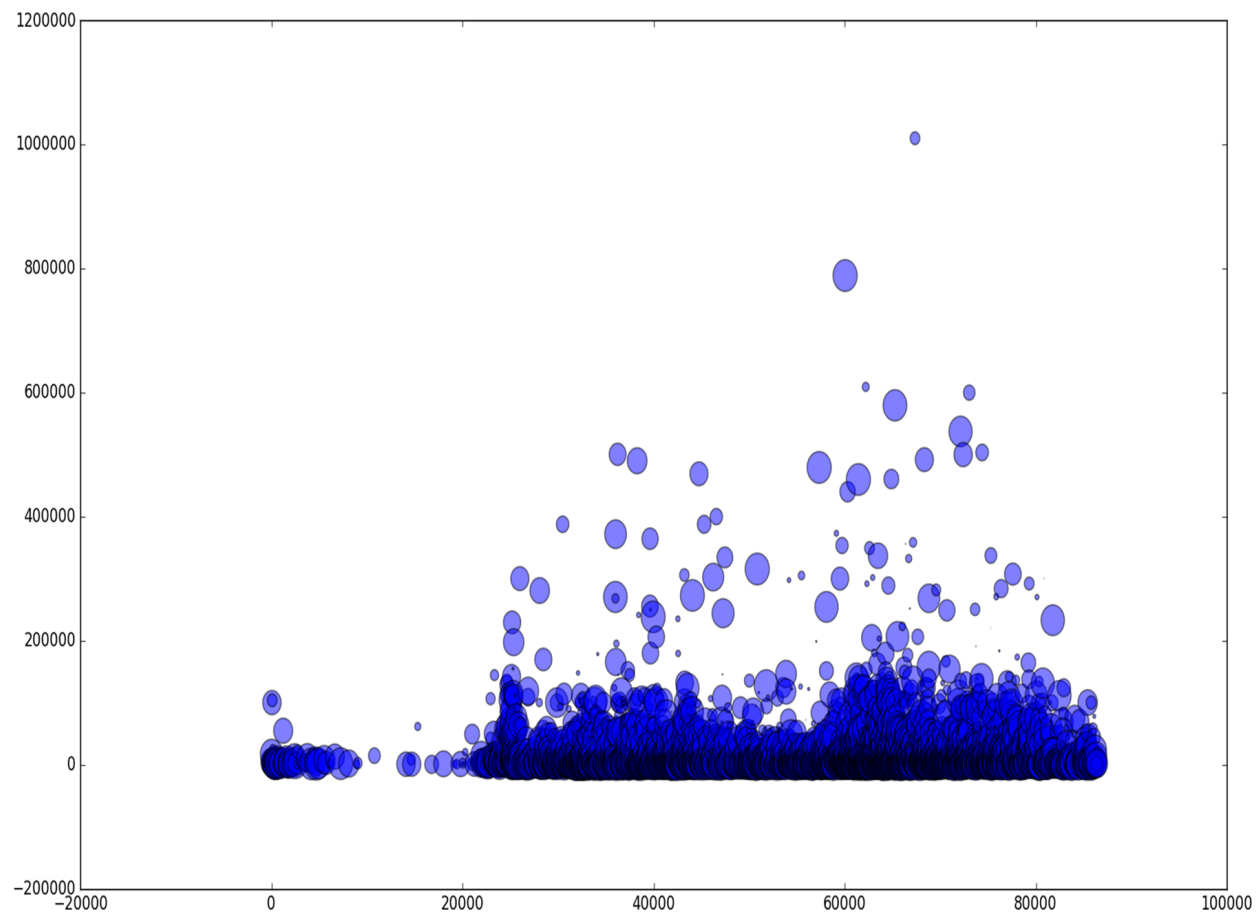

Figure 6. Subscription relations network based on WeChat comment fans

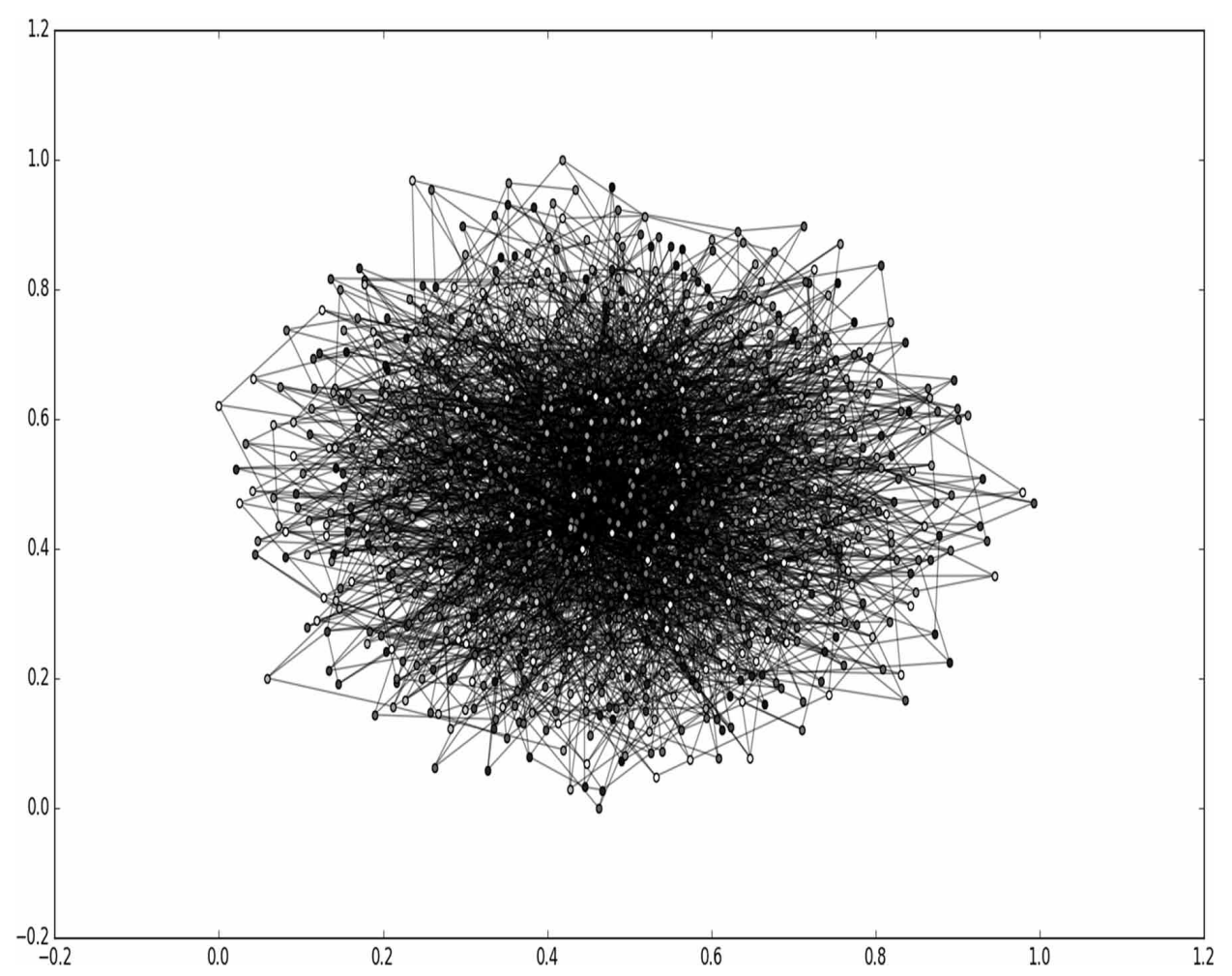


Table 6. WeChat subscription's article data and TIPN value

\begin{tabular}{|c|c|c|c|c|c|c|}
\hline \multirow[t]{2}{*}{ subscription ID } & \multicolumn{6}{|c|}{ subscription Data } \\
\hline & $\begin{array}{l}\text { Posting Time } \\
\text { Period }\end{array}$ & $\begin{array}{l}\text { Original } \\
\text { Content }\end{array}$ & $\begin{array}{c}\text { Repost } \\
\text { Content }\end{array}$ & $\begin{array}{c}\text { Image \& Text } \\
\text { Proportion }\end{array}$ & $\begin{array}{c}\text { Video } \\
\text { Proportion }\end{array}$ & $\begin{array}{l}\text { TIPN } \\
\text { Value }\end{array}$ \\
\hline Sgcctop & $0: 01: 08-21: 19: 43$ & $74.71 \%$ & $25.29 \%$ & $56 \%$ & $44 \%$ & 94.64 \\
\hline Goodenergy & 7:04:06-20:51:40 & $67.39 \%$ & $32.61 \%$ & $92 \%$ & $8 \%$ & 64.66 \\
\hline Gwgsdl & $7: 21: 14-20: 08: 11$ & $50.49 \%$ & $49.51 \%$ & $98 \%$ & $2 \%$ & 76.27 \\
\hline Gas_Hydrate & $0: 04: 25-22: 05: 40$ & $43.33 \%$ & $56.67 \%$ & $100 \%$ & $0 \%$ & 53.05 \\
\hline Tushuoshen dong & $0: 09: 57-23: 23: 29$ & $35.94 \%$ & $64.06 \%$ & $93 \%$ & $7 \%$ & 87.36 \\
\hline Cnoocdaily & $0: 06: 01-23: 18: 49$ & $34.74 \%$ & $65.26 \%$ & $92 \%$ & $8 \%$ & 61.22 \\
\hline sgcc_bj & $17: 22: 22-19: 46: 23$ & $32.00 \%$ & $68.00 \%$ & $89 \%$ & $11 \%$ & 81.38 \\
\hline tsnp01 & $7: 15: 04-23: 57: 16$ & $31.06 \%$ & $68.94 \%$ & $95 \%$ & $5 \%$ & 64.39 \\
\hline Wodytwdj & $8: 41: 10-22: 11: 46$ & $30.32 \%$ & $69.68 \%$ & $94 \%$ & $6 \%$ & 65.49 \\
\hline accenture_china & $12: 11: 06-21: 54: 11$ & $27.46 \%$ & $72.54 \%$ & $93 \%$ & $7 \%$ & 69.82 \\
\hline CSG_SZ_95598 & $10: 59: 58-21: 30: 25$ & $26.78 \%$ & $73.22 \%$ & $89 \%$ & $11 \%$ & 82.74 \\
\hline CNPC-online & $9: 59: 06-23: 19: 08$ & $25.74 \%$ & $74.26 \%$ & $96 \%$ & $4 \%$ & 94.13 \\
\hline hbytsk & $15: 43: 03-22: 54: 01$ & $25.38 \%$ & $74.62 \%$ & $95 \%$ & $5 \%$ & 85.73 \\
\hline sinochemgroup & $8: 30: 30-18: 56: 56$ & $23.39 \%$ & $76.61 \%$ & $80 \%$ & $20 \%$ & 83.45 \\
\hline zggdcgdc & $6: 13: 28-22: 25: 00$ & $21.39 \%$ & $78.61 \%$ & $94 \%$ & $6 \%$ & 73.29 \\
\hline yl-energy & $0: 43: 10-22: 58: 12$ & $18.37 \%$ & $81.63 \%$ & $95 \%$ & $5 \%$ & 69.55 \\
\hline wlww-cnoc & $7: 19: 16-23: 57: 07$ & $15.56 \%$ & $84.44 \%$ & $91 \%$ & $9 \%$ & 67.07 \\
\hline dqytwx & $16: 26: 36-18: 32: 33$ & $15.23 \%$ & $84.77 \%$ & $87 \%$ & $13 \%$ & 58.79 \\
\hline dnmc_wechat & $4: 04: 54-22: 25: 16$ & $14.34 \%$ & $85.66 \%$ & $88 \%$ & $12 \%$ & 70.28 \\
\hline sdnpcnews & $7: 01: 29-22: 28: 55$ & $13.82 \%$ & $86.18 \%$ & $94 \%$ & $6 \%$ & 71.70 \\
\hline
\end{tabular}

Figure 7. WeChat subscription relations network of the same topic
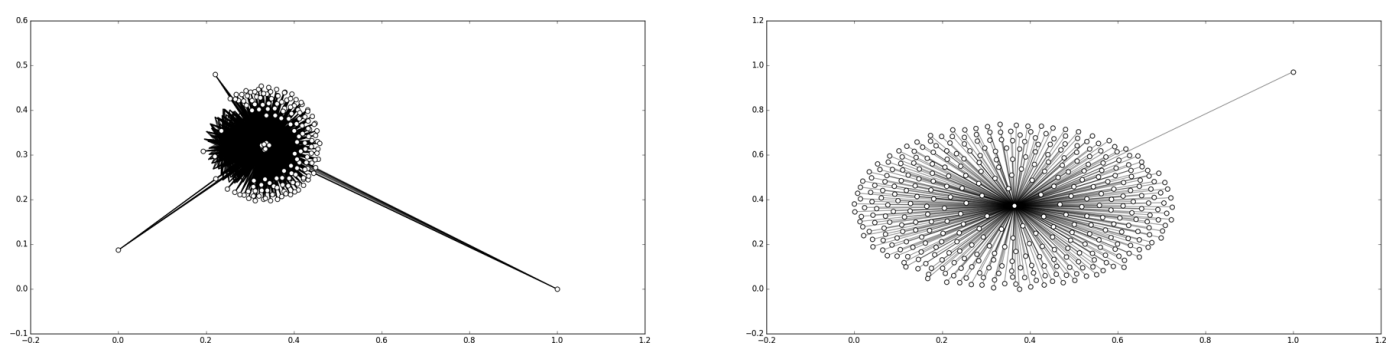
International Journal of Digital Crime and Forensics

Volume 11 • Issue 4 • October-December 2019

Figure 8. WeChat subscription relations network of distinct topic
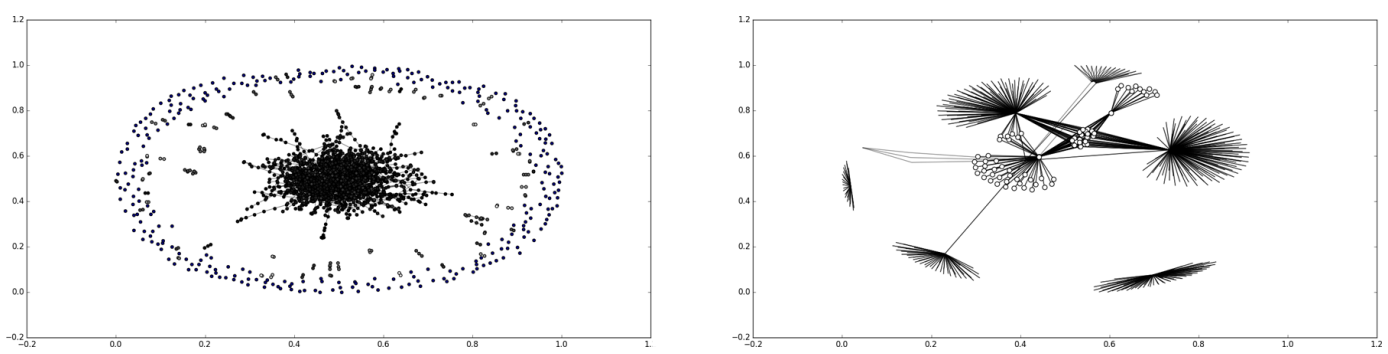


\section{REFERENCES}

Asano, Y., Yu, T., \& Nishizeki, T. (2007). Improvements of HITS algorithms for spam links. In Joint, AsiaPacific Web and, International Conference on Web-Age Information Management Conference on Advances in Data and Web Management (pp. 479-490). Springer-Verlag. doi:10.1007/978-3-540-72524-4_50

Cha, M., Haddadi, H., Benevenuto, F., \& Gummadi, K. P. (2010). Measuring user influence in twitter: the million follower fallacy.

Cha, M., Haddadi, H., Benevenuto, F., \& Gummadi, P. K. (2010). Measuring User Influence in Twitter: The Million Follower Fallacy. International Conference on Weblogs and Social Media, Icwsm 2010, Washington, $D C$ (Vol.14). DBLP.

Chen, W., Wang, Y., \& Yang, S. (2009). Efficient influence maximization in social networks. in: kdd. In Proc of Acm Kdd (pp. 199-208). doi:10.1145/1557019.1557047

Cong Zhang does his Ph.D. at Beijing University of Posts and Telecommunications, Beijing, China. His current research interests include IOT security, big data analysis.

Huang, X., Chen, J., Yan, P., \& Luo, X. (2005). Word Segmentation and POS Tagging for Chinese Keyphrase Extraction. In International Conference on Advanced Data Mining and Applications (pp. 364-369). Springer Berlin Heidelberg. doi:10.1007/11527503_44

Kempe, D., \& Kleinberg, J., \& Éva Tardos. (2003). Maximizing the spread of influence through a social network. In ACM SIGKDD International Conference on Knowledge Discovery and Data Mining (pp.137-146). ACM. doi:10.1145/956750.956769

Kleinberg, J. M. (1999). Authoritative sources in a hyperlinked environment. Journal of the Association for Computing Machinery, 46(5), 604-632. doi:10.1145/324133.324140

Kloumann, I. M., Ugander, J., \& Kleinberg, J. (2017). Block models and personalized pagerank. Proceedings of the National Academy of Sciences of the United States of America, 114(1),33-38. doi:10.1073/pnas.1611275114 PMID:27999183

Lamberti, F., Sanna, A., \& Demartini, C. (2008). A relation-based page rank algorithm for semantic web search engines. IEEE Transactions on Knowledge and Data Engineering, 21(1), 123-136. doi:10.1109/TKDE.2008.113

Li, Y., Chen, W., Wang, Y., \& Zhang, Z. L. (2011). Influence diffusion dynamics and influence maximization in social networks with friend and foe relationships.

Liao, J. H., Shi, J., Chi, S. L., \& Cheng, Y. H. (2017). Design and application of blended learning based on wechat public platform.

Liu, Y., \& Lin, Y. (2007). Supervised HITS Algorithm for MEDLINE Citation Ranking. In IEEE International Conference on Bioinformatics and Bioengineering (pp. 1323-1327). IEEE. doi:10.1109/BIBE.2007.4375740

Mao, J. X., Liu, Y. Q., Zhang, M., \& Ma, S. P. (2014). Social influence analysis for microblog user based on user behavior. Chinese Journal of Computers.

Nanchang, C. H. E. N. G., Min, H. O. U., \& Yonglin, T. E. N. G. (2015). Short text attitude analysis based on textual characteristics. Journal of Chinese Information Processing, 29(2), 163-169.

Page, L. (1998). The pagerank citation ranking: bringing order to the web. Stanford Digital Libraries Working Paper, 9(1), 1-14.

Qiu, J., Li, Y., Chen, B., Chen, B., Chen, B., \& Chen, B. (2016). The Lifecycle and Cascade of WeChat Social Messaging Groups. In International Conference on World Wide Web (pp. 311-320). International World Wide Web Conferences Steering Committee. doi:10.1145/2872427.2882979

Qiu, Y. F., Wang, L. Y., Shao, L. S., \& Guo, H. M. (2014). User interest modeling approach based on short text of micro-blog. Computer Engineering, 40(2), 275-279.

Rowe, M. (2014). SemanticSVD++: Incorporating Semantic Taste Evolution for Predicting Ratings. In IEEE/ WIC/ACM International Joint Conferences on Web Intelligence (Vol. 1, pp. 213-220). IEEE. 
Weng, J., Lim, E. P., Jiang, J., \& He, Q. (2010). Twitter rank: finding topic-sensitive influential twitterers.

Yang, C., Kefei, Y. U., Shiren, Y. E., Yan, S., Ding, H., \& Yang, J. (2012). New assessment method on influence of bloggers in community of Chinese microblog. Computer Engineering and Applications, 48(25), 229-233.

You, B., Yan, Y. S., Sun, Y. G., \& Liu, J. (2013). Method of information content evaluating semantic similarity on HowNet. Jisuanji Xitong Yingyong- Computer Systems and Applications, 22(1), 129-133.

Yuan'an Liu received his M.S. and Ph.D. degree from University of Electronic Science and technology of China 1989 in 1992, respectively. He did post-doctoral research at Carleton University, Canada, from1995-1997. He has published over 500 articles in various journals and magazines. His research interests are parallel computing, wireless sensor network technology and streaming media communication. Fan Wu received B.Sc. degree from University of Electronic Science and technology of China, Chengdu, China, in 2004, and Ph.D. degree from Beijing University of Posts and Telecommunications, Beijing, China, in 2009. Her current research interests include Privacy data protection, information security for mobile phones

Zhai Lidong received a Ph.D. degree from Beijing University of Posts and Telecommunications, Beijing, China, in 2009. His current research interests include cyberspace security, big data analysis. Lu Hui received Ph.D. degree from Beijing University of Posts and Telecommunications, Beijing, China, in 2010. His current research interests include IOT security, information security for mobile phones. 